\title{
Immunogenicity of multi-epitope-based vaccine candidates administered with the adjuvant Gp96 against rabies
}

\author{
Yange $\mathrm{Niu}^{1,2}, \mathrm{Ye} \mathrm{Liu}^{3}$, Limin Yang ${ }^{1}$, Hongren $\mathrm{Qu}^{1}$, Jingyi Zhao ${ }^{4}$, Rongliang $\mathrm{Hu}^{3}$, Jing $\mathrm{Li}^{1,5}$, \\ Wenjun Liu ${ }^{1,2}$
}

1. Key Laboratory of Pathogenic Microbiology and Immunology, Institute of Microbiology, Chinese Academy of Sciences, Beijing 100101, China

2. University of Chinese Academy of Sciences, Beijing 100049, China

3. Laboratory of Epidemiology and Key Laboratory of Jilin Province for Zoonosis Prevention and Control, Veterinary Research Institute, Academy of Military Medical Sciences, Changchun 130122, China 4. Beijing Center for Animal Disease Control and Prevention Chinese Veterinary Medical Association of Animal Clinic Branch, Beijing 102629, China

5. State Key Laboratory of Veterinary Biotechnology, Harbin Veterinary Research Institute, Chinese Academy of Agricultural Sciences, Harbin 150001, China

Rabies, a zoonotic disease, causes $>\mathbf{5 5 , 0 0 0}$ human deaths globally and results in at least $\mathbf{5 0 0}$ million dollars in losses every year. The currently available rabies vaccines are mainly inactivated and attenuated vaccines, which have been linked with clinical diseases in animals. Thus, a rabies vaccine with high safety and efficacy is urgently needed. Peptide vaccines are known for their low cost, simple production procedures and high safety. Therefore, in this study, we examined the efficacy of multi-epitope-based vaccine candidates against rabies virus. The ability of various peptides to induce epitope-specific responses was examined, and the two peptides that possessed the highest antigenicity and conservation, i.e., AR16 and hPAB, were coated with adjuvant canineGp96 and used to prepare vaccines. The peptides were prepared as an emulsion of oil in water (O/W) to create three batches of bivalent vaccine products. The vaccine candidates possessed high safety. Virus neutralizing antibodies were detected on the day 14 after the first immunization in mice and beagles, reaching $5-6 \mathrm{IU} / \mathrm{mL}$ in mice and $7-9 \mathrm{IU} / \mathrm{mL}$ in beagles by day 28 . The protective efficacy of the vaccine candidates was about $70 \%-80 \%$ in mice challenged by a virulent strain of rabies virus. Thus, a novel multi-epitope-based rabies vaccine with Gp96 as an adjuvant was developed and validated in mice and dogs. Our results suggest that synthetic peptides hold promise for the development of novel vaccines against rabies.

\section{KEYWORDS rabies virus; multi-epitope-based vaccine; immunogenicity evaluation; Gp96}

Received: 29 January 2016, Accepted: 16 March 2016

Published online: 6 April 2016

$\triangle$ Correspondence:

Jing Li, Phone: +86-10-64807503, Fax: +86-10-64807503,

Email: lj418@163.com

ORCID: 0000-0001-9588-5291

Wenjun Liu, Phone: +86-10-64807497, Fax: +86-10-64807503,

Email: liuwj@im.ac.cn

ORCID: 0000-0002-1590-7380

\section{INTRODUCTION}

Rabies is a zoonotic disease with an extremely high mortality rate of nearly $100 \%$ (Dietzschold et al., 2008). It presents as acute encephalomyelitis caused by rabies virus (RABV) in the central nervous system. RABV is a type species of the Lyssavirus genus of the Rhabdoviridae family. These viruses are enveloped and have a negative single-stranded RNA genome. The RABV genome is 
approximately $12 \mathrm{~kb}$ in length and encodes five structural proteins, including the nucleoprotein $(\mathrm{N})$, the phosphorylated protein $(\mathrm{P})$, the matrix protein $(\mathrm{M})$, the external surface glycoprotein $(\mathrm{G})$, and a RNA-dependent RNA polymerase (L) (Pulmanausahakul et al., 2008) .

Stray dogs and wild animals are natural reservoirs of RABV. Vaccination is an effective approach for preventing the spread of RABV in both humans and animals (Kaur et al., 2015). At present,commercial vaccines against RABV are mainly inactivated and attenuated vaccines. However, vaccines used in animals have been linked with clinical diseases (Takayama-Ito et al., 2006). Thus, the development of a rabies vaccine with high safety and efficacy is urgently needed (Rupprecht and Gibbons, 2004).

Peptide vaccines have drawn attention owing to their low cost, simple production procedures, and high safety (Houimel and Dellagi, 2009). Two conserved sequences of the mature glycoprotein of RABV are recognized by glycoprotein-specific antibodies based on a screening analysis of a phage-display library, and synthetic peptides that simulate these epitopes induce an epitope-specific response post-immunization in rabbits and mice (Mansfield et al., 2004). The N protein is also an important antigen of RABV. Two sequences at positions 22-168 (N-terminal) and 262-450 (C-terminal) of $\mathrm{N}$ have been confirmed as mimic epitopes by enzymelinked immunosorbent assay (ELISA) (Yang et al., 2013) .

Owing to their small molecular weight and low immunogenicity, immunization with synthetic peptides does not effectively evoke immune responses. Therefore, adjuvants are needed to increase antigen-specific immune responses (McCluskie and Weeratna, 2001; Degen et al., 2003). Many adjuvants are widely used in human and animal vaccines, such as aluminum salts (Li et al., 2007), emulsions (Guy, 2007), and chemokines (Kundi, 2007). The heat shock protein Gp96, which associates with antigenic peptides from viruses and bacteria, effectively presents antigens to both MHC class I and MHC class II molecules to activate specific T cells (Li et al., 2011). Thus, Gp96 has been applied as an adjuvant in many DNA- or peptide-based vaccines to induce immunity against cancer and infectious diseases (Bolhassani and Rafati, 2008).

In the present study, two peptides (AR16 and hPAB) induced effective antibody production. The physical properties and safety of the multi-epitope-based vaccine candidates administered with the Gp96 adjuvant were examined. The neutralizing antibody levels of immunized mice and beagles were investigated, and the protective efficacy of the vaccine candidates was monitored in mice.

\section{MATERIALS AND METHODS}

\section{Viruses, animals, and strains}

RABV, challenge virus standard 24 (CVS 24), and challenge virus standard 11 (CVS 11) were kindly provided by Prof. Rongliang Hu (Veterinary Research Institute, Academy of Military Medical Sciences, Changchun, China). Six-month-old female beagles were purchased from the Beijing Laboratory Animal Research Center. Five-week-old female BALB/c mice and four-month-old female rabbits were purchased from Vital River Laboratory Animal Technology Co. Ltd. (Beijing, China). All animals were negative for anti-RABV antibodies. Pichia pastoris yeast X33 and Escherichia coli DH5a were maintained in our laboratory.

\section{Peptide synthesis}

According to the amino acid sequences of the RABV G protein, five peptide sequences (linear neutralizing epitopes) and one random sequence (Table 1) were synthesized by LifeTein, LLC (Somerset, NJ, USA). All synthesized peptides were conjugated to keyhole limpet

Table 1. Comparison of mice immunized with various epitopes and the random peptide

\begin{tabular}{|c|c|c|c|c|c|}
\hline Groups & $\begin{array}{l}\text { Immunized } \\
\text { mice }(n)\end{array}$ & $\begin{array}{l}\text { Positive } \\
\text { number }(\mathrm{n})\end{array}$ & $\begin{array}{l}\text { Positive } \\
\text { rate (\%) }\end{array}$ & Sequence & Reference(s) \\
\hline AR16 & 20 & 19 & 95 & LVNLHDFR & $\begin{array}{l}\text { Dietzschold et al., } 1990 \\
\text { Niederhauser et al., } 2008 \\
\text { Cai et al., } 2010\end{array}$ \\
\hline MFn & 20 & 17 & 85 & LGPWSPIDIHHLSC & Mansfield et al., 2004 \\
\hline RG719 & 20 & 15 & 75 & $\begin{array}{l}\text { CTKWCPPDQLVNLH } \\
\text { DFRSDEIEHLVVEE }\end{array}$ & Ni et al., 1995 \\
\hline Peptides & 20 & 0 & 0 & SDEISTPIDG & - \\
\hline PBS & 20 & 0 & 0 & - & - \\
\hline
\end{tabular}


hemocyanin $(\mathrm{KLH})$ via a C-terminal cysteine residue. The purity of the peptides was $>95 \%$, as determined by high-performance liquid chromatography.

\section{Immunization of mice and serum isolation}

The mice were randomly separated into seven groups (n $=20 \mathrm{mice}$ (group). Six groups of BALB/c mice were immunized with $50 \mu \mathrm{g}$ of synthesized peptides each. One group was immunized with phosphate-buffered saline (PBS) as a negative control. Mice in each group were injected three times at 0,2 , and 4 weeks. The mice were sacrificed 2 weeks after the last immunization. Whole blood was isolated and incubated for $30 \mathrm{~min}$ at $37^{\circ} \mathrm{C}$, then transferred to $4{ }^{\circ} \mathrm{C}$ for $2 \mathrm{~h}$. Serum was obtained after centrifugation at $2500 \times \mathrm{g}$ for $10 \mathrm{~min}$.

\section{ELISA}

The 96-well ELISA plates were coated with $0.1 \mu \mathrm{g}$ of synthesized peptides for $1 \mathrm{~h}$ at $37{ }^{\circ} \mathrm{C}$. The plates were washed three times with PBST and blocked with blocking buffer $\left(3 \%\right.$ BSA in PBS) for $1 \mathrm{~h}$ at $37^{\circ} \mathrm{C}$. After they were washed three times with PBST, the mouse serum samples were diluted in blocking buffer and added to the wells at different dilutions, followed by incubation for 1 h at $37^{\circ} \mathrm{C}$. After three washes, $100 \mu \mathrm{L}$ of horseradish peroxidase-conjugated goat anti-mouse $\operatorname{IgG}$ was added to each well and incubated for $1 \mathrm{~h}$ at $37^{\circ} \mathrm{C}$. After washing with PBST, $100 \mu \mathrm{L}$ of the substrate solution TMB was added, and the plates were incubated for $15 \mathrm{~min}$ in the dark. The addition of $50 \mu \mathrm{L}$ of $1 \mathrm{~mol} / \mathrm{L} \mathrm{H}_{2} \mathrm{SO}_{4}$ was used to stop the reactions. The absorbance was read at $450 \mathrm{~nm}$ using an ELISA reader. Samples with a ratio of $\mathrm{OD}_{450}$ for the immunized sample to that of the negative sample of greater than 2.1 (statistic of known positive samples and negative samples) were considered positive.

\section{Construction of the canine-Gp96 recombinant expression vector}

Total canine RNA was extracted using TRIzol reagent. cDNA was obtained with AMV reverse transcriptase. The amino acids at positions 22-355 (N-terminal) of Gp96 were amplified via RT-PCR. The primers were designed according to the $H s p 90 B 1$ mRNA sequence (GenBank No. U01153.1). The amplified genes were cloned into the Xho I and $X b a$ I sites of the pPICZ $\alpha \mathrm{A}$ vector, resulting in $\mathrm{pPICZ} \alpha \mathrm{A} / \mathrm{Gp} 96 \mathrm{~N}$. The recombinant plasmid was transformed into $E$. coli $\mathrm{DH} 5 \alpha$ and identified by sequencing.

\section{Expression of the recombinant protein}

The plasmid was linearized by Dra I and transformed into $P$. pastoris $\mathrm{X} 33$ by electroporation. The positive transformants were selected in YPD medium at $30{ }^{\circ} \mathrm{C}$. Protein expression was then induced by $1 \%$ methanol. A 1 -
$\mathrm{mL}$ aliquot of culture fluid was taken from the medium on days $0,1,2,3,4$, and 5 . Proteins were purified through a His-tagged affinity column and then dialyzed in $50 \mathrm{mmol} / \mathrm{L}$ Tris- $\mathrm{HCl}$ buffer to remove unwanted small molecules. Protein expression levels were tested by western blotting.

\section{Western blotting analysis}

Protein samples were separated by $12 \%$ SDS-PAGE (sodium dodecyl sulfate polyacrylamide gel electrophoresis). Gels were transferred to a polyvinylidene difluoride membrane. Blots were saturated with $5 \%$ nonfat dried milk in TBST for $2 \mathrm{~h}$ and washed three times with TBST. Mouse anti-His monoclonal antibody (Beyotime, Beijing, China) and horseradish peroxidase-conjugated goat anti-mouse IgG (Abgent, San Diego, California, USA) were used, respectively, as the primary and secondary antibodies at the dilutions recommended by the manufacturers. Proteins were detected using a gel imaging system (ChemiScope 3400, Clinx Science Instruments Co., Ltd., Shanghai, China).

\section{Antigenicity and conservation analysis of AR16}

To assess the antigenicity and conservation of AR16, G protein sequences of $105 \mathrm{RABV}$ isolates were analyzed using DNAStar and WebLogo (Crooks et al., 2004). All settings used for these analyses were default.

\section{Vaccine candidate preparation}

The synthesized peptides AR16 and hPAB, conjugated to $\mathrm{KLH}$, were dissolved in PBS ( $\mathrm{pH}$ 7.4) to a concentration of $1.0 \mathrm{mg} / \mathrm{mL}$. The concentration of the recombinant protein Gp96 was determined using the BCA Protein Assay Kit (TIANGEN Biotech (Beijing) Co., Ltd., Beijing, China). The dissolved peptides were mixed with adjuvant Gp96 and emulsified with mineral oil to prepare the vaccine candidates. The concentrations of the peptides and Gp96 in the candidate vaccines were as follows, AR16 $250 \mu \mathrm{g} / \mathrm{mL}$, hPAB $250 \mu \mathrm{g} / \mathrm{mL}$, and Gp96 $500 \mu \mathrm{g} / \mathrm{mL}$. The vaccine candidates were qualified and packaged into three batches, LW2014001, LW2014002, and LW2014003.

\section{Physical properties of the peptide vaccine candidates}

The physical properties of the three vaccine batches were assessed in our laboratory. Appearance, viscosity, dosage form (type), sterility, formaldehyde residue, thimerosal content, and stability were analyzed.

\section{Safety analysis of the peptide vaccine candidates}

The safety of vaccines was evaluated according to the guidelines established in the Veterinary Pharmacopoeia 
of the People's Republic of China (Commission of Chinese Veterinary Pharmacopoeia, 2010). Ten BALB/c mice (5 weeks old, female), six rabbits (4 months old, female), and six beagles ( 5 months old, female) were injected with $0.4,0.4$, and $1.0 \mathrm{~mL}$ of peptide vaccine candidate, respectively, at multiple subcutaneous sites in the abdomen, back, and muscles. Animals were observed for 14 days, and local reactions and clinical symptoms were recorded.

\section{Vaccination of mice and beagles}

In the mouse model experiment, $70 \mathrm{BALB} / \mathrm{c}$ mice were $\mathrm{r}$ and omly divided into seven groups (10 mice per group). Six groups were intradermally injected with either 0.4 $\mathrm{mL}$ of three batches of peptide vaccine candidates (LW2014001, LW2014002, and LW2014003), a negative control (random peptides), the selected peptides $(\mathrm{AR} 16+\mathrm{hPAB})$, or a commercial vaccine $\left(\operatorname{Rabvac}^{\mathrm{TM}} 3\right)$. Another group was intradermally injected with an equal volume of PBS. After 14 days, booster immunization was performed via the same immunization procedures. Serum samples from the mice were collected for a neutralizing antibody analysis at days 14 and 28 after the first immunization.

In the beagle model experiment, seven groups of beagles (10 beagles per group) were intradermally injected two times at 14-day intervals with $1.0 \mathrm{~mL}$ of three batches of peptide vaccine candidates (LW2014001, LW2014002, and LW2014003), a negative control (random peptides), the selected peptides (AR16 + hPAB), a commercial vaccine (Vaccine-M, Rabvac ${ }^{\mathrm{TM}} 3$ ), or PBS. Serum samples from the beagles were collected at days 14 and 28 after the first immunization to analyze the neutralizing antibody titers.

\section{Fluorescent antibody virus neutralization (FAVN)}

FAVN was performed to determine serum virus-neutralizing antibody (VNA) levels as previously described (Cliquet et al., 1998). Briefly, $0.1 \mathrm{~mL}$ of serial threefold dilutions of the positive and negative sera and of the tested serum samples was added to 96-well plates. Then, $0.05 \mathrm{~mL}$ of CVS 11 with a $100 \mathrm{TCID}_{50}$ was added to each well containing the serum dilution. The plates were incubated for $1 \mathrm{~h}$ at $37^{\circ} \mathrm{C}$ in an incubator with $5 \% \mathrm{CO}_{2}$. The BHK-21 cells were diluted to a concentration of $4 \times$ $10^{5}$ cells $/ \mathrm{mL}$. Next, $0.05 \mathrm{~mL}$ of the cell suspension was added to each well, and the plates were incubated for 48 $\mathrm{h}$ at $37^{\circ} \mathrm{C}$. After incubation, the medium was discarded, and the cells were fixed in $80 \%$ cold acetone for $30 \mathrm{~min}$. The plates were stained by adding FITC-conjugated antirabies antibody for $30 \mathrm{~min}$ at $37^{\circ} \mathrm{C}$ and examined by fluorescence microscopy after washing with PBST. The highest dilution showing no fluorescence was calculated as the endpoint, and the data were converted to $\mathrm{IU} / \mathrm{mL}$ with the Spearman-Kärber formula according to the WHO anti-RABV antibody standard (World Organisation for Animal Health, 2013). VNA titers of rabies of $>$ $0.5 \mathrm{IU} / \mathrm{mL}$ were defined as positive.

\section{Challenge of mice with CVS 24}

All of the mice were anesthetized with ether 14 days after the booster immunization. Then, $0.03 \mathrm{~mL}$ of 100 $\mathrm{LD}_{50}$ CVS 24 was inoculated into the mouse brains. Clinical signs of rabies and mouse survival were monitored and recorded daily for 14 days.

\section{Statistical analysis}

Statistical analyses were performed and graphical presentations were obtained using GraphPad Prism software. All data are expressed as means \pm standard deviation (SD). Comparisons between groups were evaluated by Student's $t$-tests. $P$-values were considered statistically significant when they were less than 0.05 and highly significant when they were less than 0.01 .

\section{RESULTS}

\section{Screening of synthetic peptide sequences}

Mice were immunized with five peptides conjugated to $\mathrm{KLH}$ to investigate which peptide(s) effectively induce epitope-specific responses. The serum IgG antibodies in the mice were measured using ELISA. Two peptides, AR16 and hPAB, effectively induced the production of antibodies with a positive rate of $95 \%$ in mice (Table 1 ). Therefore, vaccines were prepared with AR16 and hPAB. The antigenicity index was analyzed using DNAstar software, the high antigenic regions were found in AR16 (above zero-reticle). Conservations of five amino acids in AR 16 were $100 \%$ and conservations of two amino acids in AR16 were over 98\%. Thus, AR16 was highly conserved (Figure 1), which was consistent with previous results (Cai et al., 2010).

\section{Expression of the recombinant protein canine- Gp96}

Highly expressed clones of canine-Gp96 were induced by methanol, and samples were collected at days $0,1,2,3,4$, and 5. Canine-Gp96 in sample supernatants was purified through a His-tagged affinity column and detected by western blotting (Figure 2). The expression of canineGp96 was detected at day 1 and peaked at day 4 after induction. Two bands appeared at the expected position of the target protein in the western blotting analysis because the recombinant proteins expressed in yeast were modified by glycosylation. As shown in Figure 2, we observed that canine-Gp96 reached a purity of $>85 \%$. 


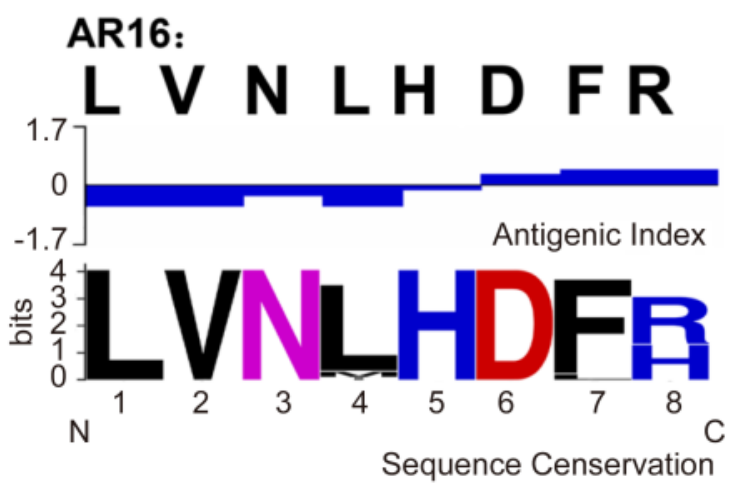

Figure 1. Antigenic characteristics and sequence conservation analyses of the peptides. The peptide from RABV glycoprotein was selected, and the antigenic index was analyzed using DNAStar software. Sequence conservation was analyzed using WebLogo. The overall height of the stack indicates the sequence conservation at the position, while the height of symbols within the stack indicates the relative frequency of each amino acid at that position.

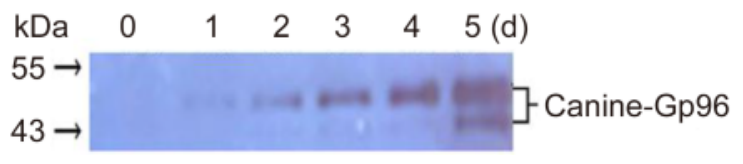

Figure 2. Expression analysis of Canine-Gp96. CanineGp96 expression was induced by methanol, and protein samples were collected for 5 days. Canine-Gp96 expression levels were assessed by western blotting.

\section{Physical properties of the peptide vaccine candidates}

A series of indices (Table 2) were assessed to characterize the physical properties of three batches of peptide vaccine candidates. The vaccine candidates were uniform milky emulsions of $\mathrm{O} / \mathrm{W}$. The viscosity of the vac- cine candidates was $\sim 7.6 \mathrm{~s} / 0.4 \mathrm{~mL}$. Formaldehyde residue and thimerosal content were at very low levels. No bacteria and no de-emulsification were detected in any batch of vaccine candidate. Thus, the vaccine candidates tested in our laboratory met the Chinese Requirements for Biologics and could be further used in animal experiments.

\section{Safety evaluation of the peptide vaccine candidates}

$\mathrm{BALB} / \mathrm{c}$ mice, rabbits, and beagles were injected with $0.4,0.4$, and $1.0 \mathrm{~mL}$ of peptide vaccine candidates, respectively, to verify the safety of the four batches of vaccines. Local reactions and clinical symptoms were recorded for 14 days. The immunized animals displayed no redness, swelling, scleroma, or clinical symptoms of rabies (data not shown). This demonstrated that the vaccine candidates were satisfactorily safe.

\section{Neutralizing antibody responses in mice and beagles}

We performed FAVN assays to determine VNA titers in the serum of mice and beagles to investigate whether the epitope vaccine candidates could induce epitope-specific immune responses against rabies. As shown in Figure 3, the VNA titers of the sera notably increased after the first immunization in the vaccine candidate groups of mice (Figure 3A) and beagles (Figure 3B). After the booster immunization, the serum VNA titers reached $\sim 5$ and 9 $\mathrm{IU} / \mathrm{mL}$ in the vaccine candidates groups of mice and beagles, respectively. Furthermore, the VNA titers of the sera in the selected peptide groups of mice and beagles were lower than those of the vaccine candidate groups after the first and booster immunizations. The difference between them was statistically significant. In addition, no VNA titers could be detected in the sera of the mice immunized with PBS and peptide controls.

Table 2. Physical properties of three batches of peptide vaccine candidate

Items

Vaccine batches

LW2014001 LW2014002 LW2014003

\begin{tabular}{llll}
\hline Appearance & Uniform milky emulsion & Uniform milky emulsion & Uniform milky emulsion \\
Viscosity (s/0.4 mL) & 7.7 & 7.6 & 7.6 \\
Dosage forms (type) & ${ }^{a} \mathrm{O} / \mathrm{W}$ & $\mathrm{O} / \mathrm{W}$ & $\mathrm{O} / \mathrm{W}$ \\
Sterility test & No bacterial growth & No bacterial growth & No bacterial growth \\
Formaldehyde residue & $0.160 \%$ & $0.158 \%$ & $0.154 \%$ \\
Thimerosal content & $0.0049 \%$ & $0.0050 \%$ & $0.0048 \%$ \\
Stability & No de-emulsification & No de-emulsification & No de-emulsification \\
\hline
\end{tabular}

Notes: ${ }^{a}: \mathrm{O} / \mathrm{W}$, oil in water. 


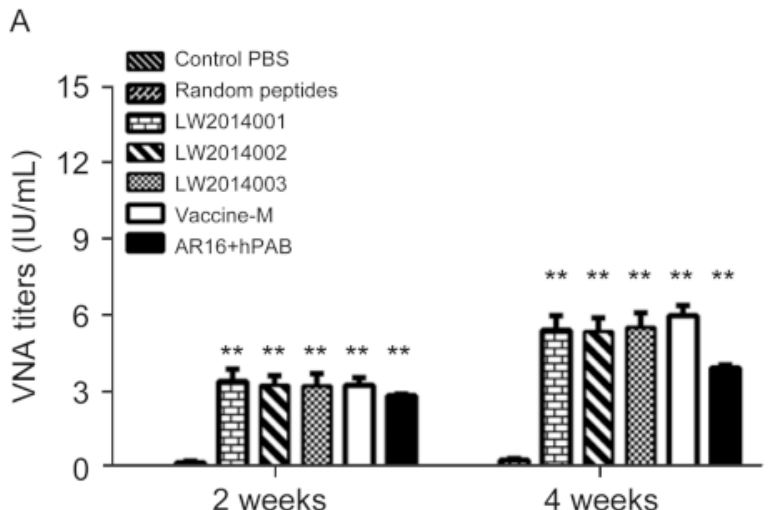

B

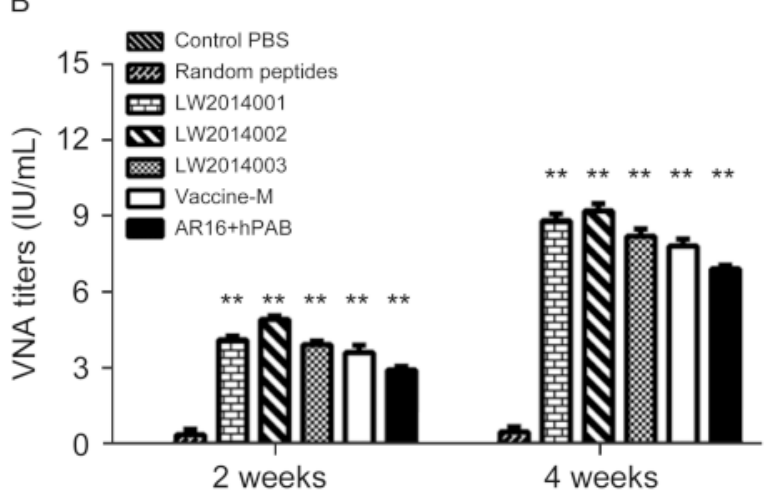

Figure 3. Specific anti-RABV antibody responses induced by peptide vaccine candidates. Mice $(A)$ and beagles $(B)$ were immunized with three batches of the peptide vaccine candidates (LW2014001, LW2014002, and LW2014003), a negative control (Random peptides), the selected peptides (AR16 + hPAB), and a commercial vaccine (Rabvac ${ }^{\mathrm{TM}} 3$ ). Serum was collected at 2 and 4 weeks after the first vaccination, and the VNA titers were measured by FAVN. ${ }^{* *}, P<0.01$.

\section{Challenge study in mice}

All tested mice were challenged with CVS 24 virus at 2 weeks after the last immunization, and the survival rate was monitored daily for 14 days (Figure 4). By day 7, we found that mice in the PBS and peptide control groups began to show clinical signs of rabies and were dead within 3 days. The mice exhibited a high survival rate (seven mice in LW2014001, eight mice in LW2014002, and eight mice in LW2014003) in the peptide vaccine candidate groups. Therefore, the protective efficacy of the peptide vaccine candidates was $70 \%-80 \%$. Furthermore, half of the mice in the selected peptides group were protected after inoculation. Only one mouse died when challenged with CVS 24 in the vaccine-M group.

\section{DISCUSSION}

When pathogenic microorganisms invade hosts, they

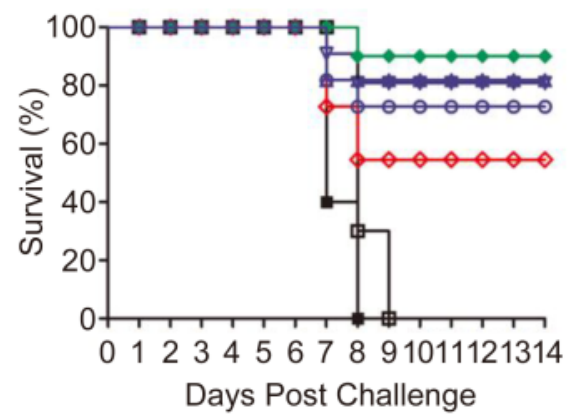

- Control PBS

$\because$ Random peptides

$\rightarrow$ Vaccine-M

- AR16+hPAB

๑ LW2014001

¿ LW2014002

$\rightarrow$ LW2014003

Figure 4. Protective efficacy of the peptide vaccine candidates against CVS 24 in mice. All tested mice were challenged with CVS 24 at two weeks after the last immunization. Survival of mice in each group was monitored and recorded daily for 14 days. Survival rate is expressed as the percentage of mice surviving in each group at different times post-challenge.

cause damage to the host body, and the hosts remove pathogens by inducing immune responses. These immune responses are not triggered by all exogenous substances, but rather by certain epitopes (Villarreal-Ramos, 2009; Yang et al., 2013). The RABV G protein is the main antigenic protein, containing more than one antigenic epitope (Marissen et al., 2005; Houimel and Dellagi, 2009; Tao et al., 2013). Previous research has shown that a recombinant pseudorabies virus expressing RABV G elicits an effective host immune response; antibody titers are maintained for $>6$ months, but are lower compared with those induced by recombinant canine adenovirus (Yuan et al., 2008). A recombinant RABV that encodes two copies of $\mathrm{G}$ protein induces higher levels of VNA and confers better protection against virulent RABV in mice and dogs than the inactivated parental virus (Liu et al., 2014; Xue et al., 2014). A candidate vaccine expressing a trimeric $G$ protein is superior to candidate vaccines that express the monomeric form, both in terms of immunogenicity and efficacy (Koraka et al., 2014).

These reports suggest that effective immune responses against rabies are induced by multiple epitopes. However, no synthetic epitope-based vaccine against RABV infection is currently available. Our results using mice and beagles revealed that multiple epitopes mixed with Gp96 can likely be used as an effective animal vaccine to prevent rabies.

Peptide vaccines containing the minimal set of key elements and more than one antigenic site induce effective immune responses (Meloen et al., 2001). Owing to the stability, ease of preparation, and high degree of specificity, the clinical application of peptide vaccines will significantly reduce the cost of prevention and treatment. 
Here, the AR16 and hPAB epitopes induced effective immune responses with a positive rate of $\sim 95 \%$. Although the other epitopes investigated (i.e., CR57, MFn, and RG719) induced the production of antibodies, their efficiencies were lower than those of AR 16 and hPAB (Table 1). These results may be explained by the fact that CR57, MFn, and RG719 induce effective immune responses only when mixed with other epitopes or in a specific conformation (Niederhauser et al., 2008).

The mice immunized with peptide vaccine candidates based on AR16 and hPAB produced specific anti-RABV antibodies after the first immunization and booster immunization (Figure 3A). The VNA titers in serum were $>$ $0.5 \mathrm{IU} / \mathrm{mL}$ and had a protective effect against RABV. The immune responses of mice between the three batches of vaccine candidates were stable and equivalent to that of the commercial vaccine (vaccine-M). In the beagle model experiment (Figure 3B), the VNA titers were higher than those in the mice 4 weeks after the first immunization. These data suggest that peptide vaccine candidates provoked strong immune responses in both mice and dogs. Nevertheless, the peptide vaccine candidates did not protect all mice challenged with CVS 24 (Figure 4), which may be due to the relatively low avidity of the antibodies. Moreover, the longevity of the induced protective response is unknown and whether the immunogenicity of vaccine candidates can be further strengthened by increasing emulsification or by mixing with highly specific epitopes remains to be studied.

The induction of protective immune responses involves antigen uptake, processing by antigen-presenting cells, activation of antigen-presenting cells for effective $\mathrm{T}$ cell priming, and activation of $\mathrm{B}$ cells (Bachmann and Jennings, 2010). Compounds that have a combination of both immunomodulatory and delivery properties can act as adjuvants (Dey and Srivastava, 2011). Freund's adjuvant, which is widely used in vaccines, is easily absorbed. However, the cost of producing it is very high. Oil adjuvant is easy to prepare and cheap, but exhibits low absorption. Thus, new kinds of adjuvants need to be evaluated. Flagellin and the Toll-like receptor 3 agonist PIKA have been investigated in rabies vaccines as adjuvants (Xiao et al., 2015; Zhang et al., 2016). The Gp96 protein used in our peptide vaccine candidates is natively located in the endoplasmic reticulum. It activates $\mathrm{CD} 8^{+} \mathrm{T}$ cells by presenting antigens to MHC-I molecules and induces immune responses via the NF- $\mathrm{KB}$ pathway (Wang et al., 2011). Gp96 is easily prepared and absorbed. Previous studies have demonstrated that Gp96 enhances the immunogenicity of subunit vaccines against PRRSV (Chen et al., 2012). However, Gp96 has not been used as an adjuvant in rabies vaccines. In the present study, adjuvant Gp96 mixed with peptides displayed satisfactory safety and enhanced the immunogenicity of peptide vaccine candidates (Figure 3 ). However, cellular immune responses in mice and dogs remain to be evaluated. The adjuvant Gp96 will be compared with other adjuvant, such as Freund's adjuvant and oil adjuvants in peptide vaccine candidates against rabies.

In conclusion, a novel rabies vaccine based on multiple epitopes using Gp96 as an adjuvant was developed and validated in mice and dogs. The epitopes were screened by immunization of mice with synthetic peptides. We demonstrated that the epitope vaccine candidates were safe in mice, rabbits, and beagles. Furthermore, the peptide vaccine candidates induced qualitatively better immune responses in mice and dogs and had strong protective effects in mice. These data provide important information for understanding the immune responses initiated by peptide vaccine candidates after challenges with RABV. Moreover, Gp96 can be used as an attractive adjuvant to enhance antigen-specific immune responses in mammals. These data hold promise for the development of novel peptide vaccines against rabies.

\section{ACKNOWLEDGMENTS}

We thank Shuang Zhang and Xi Liang for technical and logistic service support, as well as Prof. Baoshou Yang (Tianjin Ringpu Bio-technology Co., Ltd., Tianjin, China) for comments on the manuscript. This work was supported by grants from the Special Fund for Agro-scientific Research in the Public Interest (201103032), the Fund of State Key Laboratory of Veterinary Biotechnology (SKLVBF201614), the National High Technology Research and Development Program of China (2015BAD 11B02), Key Research Program of the Chinese Academy of Sciences (KSZD-EW-Z-005-001), and the Special Fund for Chinese Academy of Sciences (CZBZX-1). WJL is the principal investigator of the NSFC Innovative Research Group (Grant No. 81321063) .

\section{COMPLIANCE WITH ETHICS GUIDELINES}

The animal research was approved by the Chinese Academy of Sciences of Research Ethics Committee, under approval number PZIMCAS2013001, and complied with the Beijing Laboratory Animal Welfare and Ethical Guidelines of the Beijing Administration Committee of Laboratory Animals.

\section{AUTHOR CONTRIBUTIONS}

$\mathrm{JL}$ and WJL are co-senior authors, who conceived and designed the research. YGN, YL, and HRQ performed the experiments. YGN, LMY, HRQ, JL, and WJL performed the data analysis, prepared the manuscript, and completed its revision. RLH and JYZ provided helpful suggestions regarding the study. All authors read and approved the final manuscript. 


\section{REFERENCES}

Bachmann MF, Jennings GT. 2010. Vaccine delivery: a matter of size, geometry, kinetics and molecular patterns. Nat Rev Immunol, 10: 787-796.

Bolhassani A, Rafati S. 2008. Heat-shock proteins as powerful weapons in vaccine development. Expert Rev Vaccines, 7: $1185-1199$.

Cai K, Feng JN, Wang Q, Li T, Shi J, Hou XJ, Gao X, Liu H, Tu W, Xiao L, Wang H. 2010. Fine mapping and interaction analysis of a linear rabies virus neutralizing epitope. Microbes Infect, 12: 948-955.

Chen C, Li J, Bi Y, Jia X, Meng S, Sun L, Liu W. 2012. Gp96 enhances the immunogenicity of subunit vaccine of porcine reproductive and respiratory syndrome virus. Virus Res, 167: 162172.

Cliquet F, Aubert M, Sagne L. 1998. Development of a fluorescent antibody virus neutralisation test (FAVN test) for the quantitation of rabies-neutralising antibody. J Immunol Methods, 212: 79-87.

Commission of Chinese Veterinary Pharmacopoeia. 2010. Veterinary Pharmacopoeia of the People's Republic of China. Chemical Industry Press: 163-168.

Crooks GE, Hon G, Chandonia JM, Brenner SE. 2004. WebLogo: a sequence logo generator. Genome Res, 14: 1188-1190.

Degen WG, Jansen T, Schijns VE. 2003. Vaccine adjuvant technology: from mechanistic concepts to practical applications. Expert Rev Vaccines, 2: 327-335.

Dey AK, Srivastava IK. 2011. Novel adjuvants and delivery systems for enhancing immune responses induced by immunogens. Expert Rev Vaccines, 10: 227-251.

Dietzschold B, Gore M, Marchadier D, Niu HS, Bunschoten HM, Otvos L, Jr., Wunner WH, Ertl HC, Osterhaus AD, Koprowski H. 1990. Structural and immunological characterization of a linear virus-neutralizing epitope of the rabies virus glycoprotein and its possible use in a synthetic vaccine. J Virol, 64: 38043809.

Dietzschold B, Li J, Faber M, Schnell M. 2008. Concepts in the pathogenesis of rabies. Future Virol, 3: 481-490.

Guy B. 2007. The perfect mix: recent progress in adjuvant research. Nat Rev Microbiol, 5: 505-517.

Houimel M, Dellagi K. 2009. Peptide mimotopes of rabies virus glycoprotein with immunogenic activity. Vaccine, 27: 46484655.

Kaur M, Garg R, Singh S, Bhatnagar R. 2015. Rabies vaccines: where do we stand, where are we heading? Expert Rev Vaccines, 14: 369-381.

Koraka P, Bosch BJ, Cox M, Chubet R, Amerongen G, LovgrenBengtsson K, Martina BE, Roose J, Rottier PJ, Osterhaus AD. 2014. A recombinant rabies vaccine expressing the trimeric form of the glycoprotein confers enhanced immunogenicity and protection in outbred mice. Vaccine, 32: 4644-4650.

Kundi M. 2007. New hepatitis B vaccine formulated with an improved adjuvant system. Expert Rev Vaccines, 6: 133-140.

Li H, Nookala S, Re F. 2007. Aluminum Hydroxide Adjuvants Activate Caspase-1 and Induce IL-1 and IL-18 Release. J Immunol, 178: 5271-5276.

Li Y, Song H, Li J, Wang Y, Yan X, Zhao B, Zhang X, Wang S, Chen L, Qiu B, Meng S. 2011. Hansenula polymorpha expressed heat shock protein gp96 exerts potent $\mathrm{T}$ cell activation activity as an adjuvant. J Biotechnol, 151: 343-349.

Liu X, Yang Y, Sun Z, Chen J, Ai J, Dun C, Fu ZF, Niu X, Guo $X .2014$. A recombinant rabies virus encoding two copies of the glycoprotein gene confers protection in dogs against a virulent challenge. PLoS One, 9: e87105.

Mansfield KL, Johnson N, Fooks AR. 2004. Identification of a conserved linear epitope at the $\mathrm{N}$ terminus of the rabies virus glycoprotein. J Gen Virol, 85: 3279-3283.
Marissen WE, Kramer RA, Rice A, Weldon WC, Niezgoda M, Faber M, Slootstra JW, Meloen RH, Clijsters-van der Horst M, Visser TJ, Jongeneelen M, Thijsse S, Throsby M, de Kruif J, Rupprecht CE, Dietzschold B, Goudsmit J, Bakker AB. 2005. Novel rabies virus-neutralizing epitope recognized by human monoclonal antibody: fine mapping and escape mutant analysis. J Virol, 79: 4672-4678.

McCluskie MJ, Weeratna RD. 2001. Novel adjuvant systems. Curr Drug Targets Infect Disord, 1: 263-271.

Meloen RH, Langeveld JP, Schaaper WM, Slootstra JW. 2001. Synthetic peptide vaccines: unexpected fulfillment of discarded hope? Biologicals, 29: 233-236.

Ni Y, Tominaga Y, Honda Y, Morimoto K, Sakamoto S, Kawai A. 1995. Mapping and characterization of a sequential epitope on the rabies virus glycoprotein which is recognized by a neutralizing monoclonal antibody, RG719. Microbiol Immunol, 39: 693-702.

Niederhauser S, Bruegger D, Zahno ML, Vogt HR, Peterhans E, Zanoni R, Bertoni G. 2008. A synthetic peptide encompassing the G5 antigenic region of the rabies virus induces high avidity but poorly neutralizing antibody in immunized animals. Vaccine, 26: 6749-6753.

Pulmanausahakul R, Li J, Schnell MJ, Dietzschold B. 2008. The glycoprotein and the matrix protein of rabies virus affect pathogenicity by regulating viral replication and facilitating cell-tocell spread. J Virol, 82: 2330-2338.

Rupprecht CE, Gibbons RV. 2004. Clinical practice. Prophylaxis against rabies. N Engl J Med, 351: 2626-2635.

Takayama-Ito M, Inoue K, Shoji Y, Inoue S, Iijima T, Sakai T, Kurane I, Morimoto K. 2006. A highly attenuated rabies virus HEP-Flury strain reverts to virulent by single amino acid substitution to arginine at position 333 in glycoprotein. Virus Res, 119: 208-215.

Tao X, Han N, Guo Z, Tang Q, Rayner S, Liang G. 2013. Molecular characterization of China human rabies vaccine strains. Virol Sin, 28: 116-123.

Villarreal-Ramos B. 2009. Towards improved understanding of protective mechanisms induced by the BCG vaccine. Expert Rev Vaccines, 8: 1531-1534.

Wang S, Qiu L, Liu G, Li Y, Zhang X, Jin W, Gao GF, Kong X, Meng S. 2011. Heat shock protein gp96 enhances humoral and $\mathrm{T}$ cell responses, decreases Treg frequency and potentiates the anti-HBV activity in BALB/c and transgenic mice. Vaccine, 29: $6342-6351$.

World Organisation for Animal Health. 2013. Manual of Diagnostic Tests and Vaccines for Terrestrial Animals. OIE Terrestrial Manual: Chapter 2.1.13.

Xiao XX, Zhang Y, Liu JX, Wei QL, Yin XP. 2015. Immunoenhancement with flagellin as an adjuvant to whole-killed rabies vaccine in mice. Arch Virol, 161: 685-691.

Xue XH, Zheng XX, Wang HL, Ma JZ, Li L, Gai WW, Wang TC, Yang ST, Xia XZ. 2014. An inactivated recombinant rabies CVS-11 virus expressing two copies of the glycoprotein elicits a higher level of neutralizing antibodies and provides better protection in mice. Virus Genes, 48: 411-420.

Yang L, Cen J, Xue Q, Li J, Bi Y, Sun L, Liu W. 2013. Identification of rabies virus mimotopes screened from a phage display peptide library with purified dog anti-rabies virus serum IgG. Virus Res, 174: 47-51.

Yuan Z, Zhang S, Liu Y, Zhang F, Fooks AR, Li Q, Hu R. 2008. A recombinant pseudorabies virus expressing rabies virus glycoprotein: safety and immunogenicity in dogs. Vaccine, 26: 1314-1321.

Zhang Y, Zhang S, Li W, Hu Y, Zhao J, Liu F, Lin H, Liu Y, Wang L, Xu S, Hu R, Shao H, Li L. 2016. A novel rabies vaccine based-on toll-like receptor 3 (TLR3) agonist PIKA adjuvant exhibiting excellent safety and efficacy in animal studies. Virology, 489: 165-172. 